\title{
Characterizing the Effectiveness of Atomic Layer Deposited Coatings for the Prevention of Glass Disease
}

\author{
Miriam E. Hiebert ${ }^{1}$, Raymond J. Phaneuf ${ }^{1}$, and Ed Vicenzi ${ }^{2}$ \\ ${ }^{1}$ University of Maryland, Department of Materials Science and Engineering, College Park, Maryland \\ 20742 USA. \\ ${ }^{2}$ Smithsonian Institution, Museum Conservation Institute, 4210 Silver Hill Rd. Suitland, Maryland \\ 20746 USA.
}

Glass disease, the broad term used to describe the process by which historic glass objects degrade, presents a particularly challenging problem for museum curators and conservators. This challenge stems from both an imprecise understanding of how glass disease progresses and a lack of treatment options to slow or stop it. This work is a joint investigation by the University of Maryland, Department of Materials Science and Engineering and the Smithsonian Institution, Museum Conservation Institute that is aimed at both testing and building upon recent findings by the glass science community about the chemistry and kinetics of glass disease, and proposing a potential treatment option.

Conventional museum practice for glass conservation is often based upon untested assumptions; as an example, a common suggestion is that the progression of object degradation due to glass disease stems from a loss of water, i.e., that "drying out" is the cause of the cracking and opacification often observed [1]. In contrast, recent work from glass science literature [2,3], as well as our own results, suggest that the opposite is, in fact, responsible for the onset and progression of glass disease. We have observed early stages of glass disease on samples that have been continuously submerged in aqueous solutions. These results indicate that effective preservation strategies must focus on preventing water from contacting the surface of the glass.

We are able to prevent the direct contact of water with the surface of glass objects through the application of a highly impermeable coating using Atomic Layer Deposition (ALD). ALD is a technique that was developed for use in microelectronics and allows for the buildup of an amorphous oxide film, one atom layer at a time. In previous work, our group has shown that ALD coatings can prevent tarnishing by acting as a diffusion barrier between silver objects and atmospheric corrosive species [4]. Benefits of this technique include atomic level uniformity of the coating regardless of object's topography, ease of removal using mild alkali solutions, and minimal impact on the object's appearance.

Early results have been obtained by comparing the structural evolution of the near-surface region of glass samples with and without ALD coatings after a series of accelerated aging/aqueous acidic immersion experiments as observed in light microscopy, and the chemical changes that occur during the ion exchange reaction that drives the process as determined via x-ray microanalysis. A significant finding in our studies is that crack formation nucleates at the interface between the corrosion layer and the underlying pristine glass, and not at the sample surface as has sometimes been previously assumed [Fig.1]. Scanning electron microscopy-based energy dispersive x-ray spectroscopy (SEM-EDS) analysis showed migration of sodium ions in the glass matrix due to acid attack. We have found direct evidence for the depletion of sodium around the cracks in the corroded gel layer, as well as precipitate products both in the cracks, and along the surface [Fig. 2]. 
Future experiments are planned for more detailed exploration of the chemical and molecular structure of the corrosion layer using spectroscopic probes to gain a better understanding of changes imposed on the structure of the glass though degradation and the degree to which these processes can be prevented through the use of applied ALD coatings.

\section{References:}

[1] S. Koob in "Conservation and Care of Glass Objects", (Archetype Publications, London) p.12-18.

[2] R. Hellmann et al, Nature Materials 14 (2015) p. 307-311.

[3] B.C. Bunker et al, Journal of Non-Crystalline Solids 179 (1994), p. 300-308.

[4] A. E. Marquardt et al, Heritage Science 3 (2015).

[5] The authors acknowledge funding from the Smithsonian Institution/University of Maryland Seed Grant program.

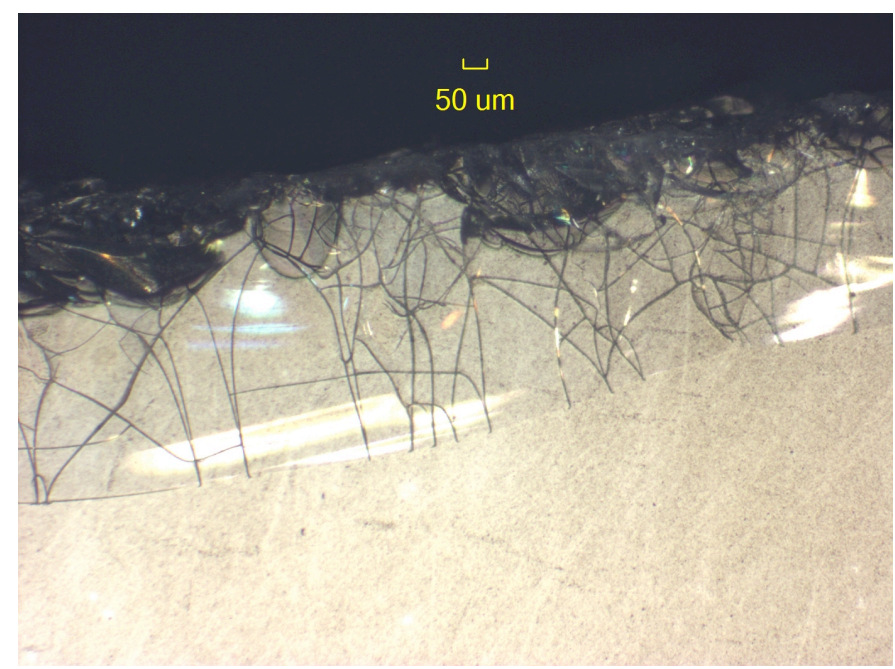

Figure 1. Hydrated gel layer and associated cracking produced by 48 hours of high temperate acid attack of a glass sample.
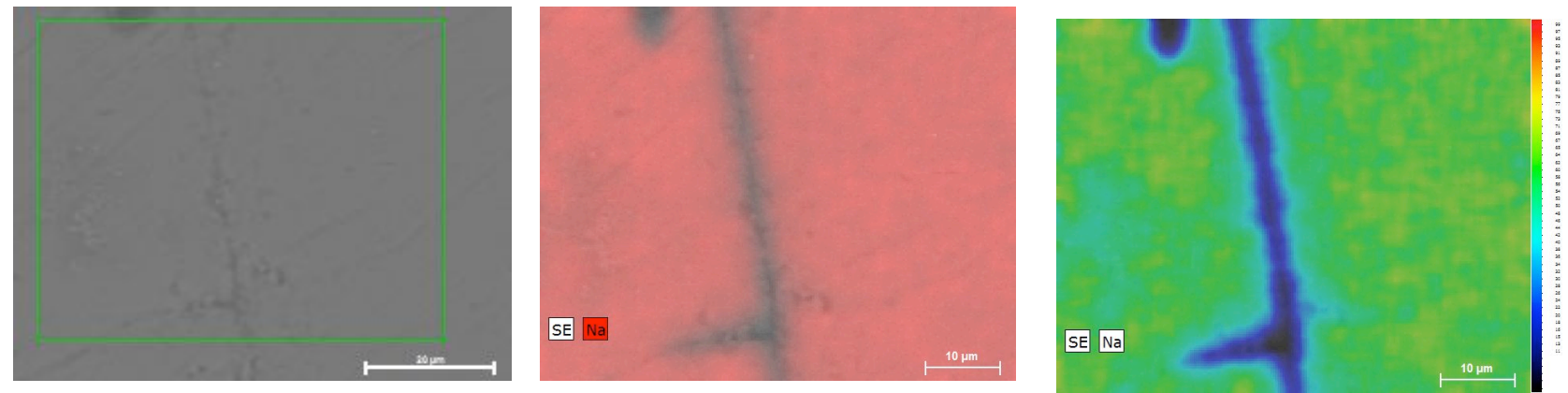

Figure 2. Left to right: SEM image of corroded layer, sodium concentration map, sodium concentration map viewed on a logarithmic scale. 